\title{
Weak G-band stars on the H-R diagram: clues to the origin of the $\mathrm{Li}$ anomaly
}

\author{
A. Palacios ${ }^{1}$, M. Parthasarathy ${ }^{2,3,4}$, Y. Bharat $\mathrm{Kumar}^{5}$, and G. Jasniewicz ${ }^{1}$ \\ 1 LUPM UMR 5299 CNRS/UM2, Université Montpellier II, CC 72, 34095 Montpellier Cedex 05, France \\ e-mail: ana.palacios@univ-montp2.fr \\ 2 National Astronomical Observatory of Japan 2-21-1 Osawa, Mitaka, Tokyo 181-8588, Japan \\ ${ }^{3}$ McDonnell Center for the Space Sciences, Physics department, Washington University in St. Louis, One Brookings Drive St. Louis, \\ MO 63130, USA \\ 4 Aryabhatta Research Institute of Observational Sciences (ARIES), 263129 Nainital, India \\ 5 Indian Institute of Astrophysics, 2nd block Koramangala, 560034 Bangalore, India
}

Received 31 August 2011 / Accepted 26 November 2011

\section{ABSTRACT}

\begin{abstract}
Aims. Weak G-band (WGB) stars are a rare class of cool luminous stars that present a strong depletion in carbon, but also lithium abundance anomalies that have been little explored in the literature since the first discovery of these peculiar objects in the early 1950 s. Here we focus on the Li-rich WGB stars and report on their evolutionary status. We explored different paths to propose a tentative explanation for the lithium anomaly.

Methods. Using archive data, we derived the fundamental parameters of WGB $\left(T_{\text {eff }}, \log g, \log \left(L / L_{\odot}\right)\right)$ using HIPPARCos parallaxes and recent temperature scales. From the equivalent widths of Li resonance line at $6707 \AA$, we uniformly derived the lithium abundances and applied NLTE corrections when possible following the procedure described by Lind et al. (2009). We also computed dedicated stellar evolution models in the mass range 3.0 to $4.5 M_{\odot}$, exploring the effects of rotation-induced and thermohaline mixing. These models are used to locate the WGB stars in the H-R diagram and to explore the origin of the abundance anomalies.

Results. The location of WGB stars in the Hertzsprung-Russell diagram shows that these are intermediate mass stars of masses ranging from 3.0 to $4.5 M_{\odot}$ located at the clump, which implies a degeneracy of their evolutionary status between subgiant/red giant branch and core helium burning phases. The atmospheres of a large proportion of WGB stars (more than 50\%) exhibit lithium abundances $A(\mathrm{Li}) \geq 1.4$ dex similar to Li-rich K giants. However, the position of WGB stars along with the Li-rich $\mathrm{K}$ giants in the $\mathrm{H}$-R diagram, indicates that both are well-separated groups. The combined and tentatively consistent analysis of the abundance pattern for lithium, carbon, and nitrogen of WGB stars seems to indicate that carbon underabundance could be decorrelated from the lithium and nitrogen overabundances.
\end{abstract}

Key words. stars: evolution - stars: abundances - stars: late-type - stars: carbon

\section{Introduction}

\section{Defining WGB stars}

The weak G-band (hereafter WGB) stars are G and $\mathrm{K}$ giants whose spectra show very weak or absent G-bands of the $\mathrm{CH} \mathrm{A}^{2} \Delta-\mathrm{X}^{2} \Pi$ system at $4300 \AA$. These stars were first identified as stellar class by Bidelman (1951), and have been mainly studied in the late 1970s and early 1980s (see e.g. for instance Sneden et al. 1978; Rao 1978; Parthasarathy \& Rao 1980; Day 1980). These stars are rare with less than 30 known to date among the population of G-K giants in the Galaxy.

Chemical composition studies (Sneden et al. 1978; Cottrell $\&$ Norris 1978) demonstrated that they are very much underabundant in carbon (typical $[\mathrm{C} / \mathrm{Fe}] \approx-1.4$ ) and present small overabundances of nitrogen and normal oxygen. Sneden et al. (1978) analyzed the ${ }^{13} \mathrm{CN}$ red-system features in the highresolution spectra of weak G-band stars and found ${ }^{12} \mathrm{C} /{ }^{13} \mathrm{C}=4$. Hartoog et al. (1977) from the photometry of the $2.3 \mu \mathrm{m} \mathrm{CO}$ vibration-rotation bands confirmed the underabundances of carbon in a large sample of weak G-band stars. The $\mathrm{CH}$ band strengths observed by Rao (1978) also indicate that the weakening of the G-band is caused by underabundance of carbon.
Sneden \& Pilachowski (1984) obtained high-resolution spectra of the $2 \mu \mathrm{m}$ first-overtone $\mathrm{CO}$ bands in the weak G-band giants and found excellent agreement between the carbon abundances derived from $\mathrm{CO}$ data and those determined using features of the CH G-band. They have found ${ }^{12} \mathrm{C} /{ }^{13} \mathrm{C} \leq 4$, which agrees with the predicted ratio for the $\mathrm{CN}$-cycle in equilibrium. The normal abundance of oxygen and sodium shows that the atmospheres of WGB stars are probably not mixed with ON-cycle processed material (Sneden et al. 1978; Drake \& Lambert 1994).

Additional chemical constraints are given by lithium and beryllium abundance determinations. Li abundances have been derived for several WGB stars and several stars were found to be lithium rich, e.g. with $A(\mathrm{Li})^{1} \geq 1.4$ (see Brown et al. 1989 ) and reaching up to $A(\mathrm{Li})=3$ (Lambert \& Sawyer 1984; Parthasarathy \& Rao 1980). Lambert \& Sawyer (1984) also report on the possible presence of ${ }^{6} \mathrm{Li}$ in HR 1299 , but the profile of the $6707 \AA{ }^{7} \mathrm{Li}$ line that is better reproduced with ${ }^{6} \mathrm{Li} /{ }^{7} \mathrm{Li} \neq 0$, could also be reproduced without having to invoke the presence of ${ }^{6} \mathrm{Li}$. Be abundances were derived from IUE spectra for three Li-rich weak G-band stars by Parthasarathy et al. (1984). Providing a differential analysis between the WGB stars and

1 With the classical notation $A(\mathrm{Li})=\log \left(N_{\mathrm{Li}} / N_{\mathrm{H}}\right)+12$. 
$\mathrm{K}$ giants in the Hyades, Be is found to be similar in both groups, and compatible within presumably large errorbars ${ }^{2}$, with the expected post dredge-up Be abundances according to standard stellar evolution models.

\section{Tentative evolutionary status and initial mass estimate}

Owing to their relative low temperature and gravity, WGB stars are classified as giants and are situated among the very crowded area of the Hertzsprung-Russell (hereafter HR) diagram populated with stars on the subgiant branch, the red giant branch (e.g. RGB), the core helium burning phase and the asymptotic giant branch (e.g. AGB). This has made the definition of their evolutionary status and initial mass difficult. However, they seem to have been unanimously qualified as core helium burning stars. In particular, Sneden et al. (1978) tentatively attribute a mass of $\approx 1 M_{\odot}$ to HR 6766 (e.g. HD 165634) and exclude it from being more massive than $3 M_{\odot}$ because of the magnitude they derive. With such a low-mass, that star should then have undergone the He flash. Let us note that Sneden et al. (1978) conjecture is based on a very crude estimate of the luminosity owing to the large error bars that impair the parallax of HR 6766. Cottrell \& Norris (1978) also identify WGB stars in their sample as being in the mass range $1.5 M_{\odot} \leq M \leq 3 M_{\odot}$ past the He flash. Subsequently, Lambert \& Sawyer (1984) proposed that WGB stars could be the progeny of magnetic Ap stars, with initial masses of 2-3 $M_{\odot}$ that have undergone a dredge-up, but they made no clear statement of their evolutionary status. More recently, Parthasarathy \& Jasniewicz $(2000,2002)$ suggested that WGB stars might be the descendants of intermediate-mass stars with $2 M_{\odot} \leq M \leq 5 M_{\odot}$. In that case, WGB would not go through the He flash, and will undergo a different evolution than that of the lower mass stars. This in turn may have an impact on the possible mechanisms that can be invoked to explain the abundance anomalies.

\section{Comparison with other carbon-depleted and Li-rich giants}

The WGB stars are therefore possibly low- to intermediate- mass evolved stars exhibiting strong depletion of carbon that can be accompanied in several cases by a Li enrichment. One may wonder how these objects compare with the other stars that also present signatures of $\mathrm{CN}$-cycle processed material in their atmospheres and exhibit unexpected Li enrichment.

Giant stars with depleted carbon and enhanced nitrogen abundances are found in almost all environments among giants. These are essentially low-mass stars on the RGB. Charbonnel \& Do Nascimento (1998) showed that $96 \%$ of field RGB stars with metallicities from solar to $1 / 1000$ solar and $M_{V} \leq-0.5$ have a carbon isotopic ratio lower than what is predicted by standard theory. In their survey of metal-poor field stars, Gratton et al. (2000) showed that the unexpected decrease of the carbon isotopic ratio occurs at the RGB bump, when the outgoing hydrogen burning shell (hereafter HBS) erases the mean molecular weight discontinuity left behind by the retreating convective envelope as a result of the first dredge-up. It is furthermore associated with a decrease of the carbon abundance $[\mathrm{C} / \mathrm{Fe}]$, an increase of the nitrogen abundance $[\mathrm{N} / \mathrm{Fe}]$ and a strong decrease of the lithium abundance, all characteristic of $\mathrm{CN}$-cycle processed material. Oxygen does not seem to be affected, as in the case of WGB stars.

\footnotetext{
2 The authors do not indicate any errorbar in the paper and give abundances of Be "rounded to the nearest 0.5 dex".
}

Typically, Charbonnel \& Do Nascimento (1998) report that around solar metallicity the lower envelope for the ${ }^{12} \mathrm{C} /{ }^{13} \mathrm{C}$ ratio in low-mass RGB stars is of about 12. Gratton et al. (2000) report $[\mathrm{C} / \mathrm{Fe}]=-0.58 \pm 0.03$ as a mean carbon abundance for upper RGB low-metallicity field stars (e.g. $\log \left(L / L_{\odot}\right)>2$ and $-2 \leq[\mathrm{Fe} / \mathrm{H}] \leq-1)$, and ${ }^{12} \mathrm{C} /{ }^{13} \mathrm{C}$ between $6-10$. These values are higher than the mean obtained for WGB stars.

In addition to that, Li-enrichment also exists among $\mathrm{K}$ giants. It is a very rare phenomenon found in less than $2 \%$ of the $\mathrm{K}$ giant stars (Kumar et al. 2011). Charbonnel \& Balachandran (2000) have shown that around solar metallicity, giants exhibiting a genuine overabundance of $\mathrm{Li}$ (e.g. excluding those giants that are still undergoing the first dredge-up) appear to cluster around the RGB bump and the clump. Very recently, however, Monaco et al. (2011) have studied Li-rich RGB stars in the Galactic thick disk, and showed that at low metallicity Li-rich giants do not cluster at the bump and are found scattered between the bump and the tip of the RGB (see also Lebzelter et al. 2011). Kumar et al. (2011) confirmed the finding of Charbonnel \& Balachandran (2000), with Li-rich stars clustered at the RGB bump, and reported some new objects that seem to be more evolved, may be past the RGB tip, and undergoing central He burning. Let us note that Li-rich $\mathrm{K}$ giant stars around the bump region do not present "anomalous" carbon isotopic ratios, e.g., the ${ }^{12} \mathrm{C} /{ }^{13} \mathrm{C}$ determined in these stars agrees well with the predictions of post dredge-up dilution (Charbonnel \& Balachandran 2000; Kumar et al. 2011).

In these $\mathrm{K}$ giants, $\mathrm{Li}$ enrichment is thought to have an internal origin (so-called Cameron \& Fowler mechanism ) and to be a short-lived phase preceding the phase of transport of CN-cycle processed material from the HBS to the surface (Charbonnel \& Balachandran 2000; Palacios et al. 2001). The extra-mixing mechanism that is capable of transporting $\mathrm{Li}$ in regions where it is preserved from proton captures and that connects the convective envelope to these regions is not clearly identified (Palacios et al. 2001; Palmerini et al. 2011).

In summary, in low-mass RGB stars, extra-mixing processes are clearly responsible for bringing up those products of the $\mathrm{CN}$-cycle that occurs in the outer layers of the HBS deeper inside the star to the stellar surface, and may be also responsible for strong and short-lived episodes of Li enrichment. The specific location of Li-rich $\mathrm{K}$ giants (see, for example Jasniewicz et al. 1999) points towards an extra-mixing process that becomes efficient only when no strong molecular weight barrier shields the $\mathrm{CN}$-cycle processed regions. This occurs only after the RGB bump in low-mass stars $\left(M \leq 2.3 M_{\odot}\right)$ or on the early AGB for stars that do not go through the He flash $\left(M>2.3 M_{\odot}\right)$. Carbon depletion and nitrogen enhancement at the stellar surface that would result from internal mixing are therefore expected to happen during these specific evolutionary stages according to the proposed self-consistent scenarios (Charbonnel \& Balachandran 2000; Charbonnel \& Lagarde 2010).

\section{Paper outline}

We propose a new re-analysis of the data available for WGB stars and investigate the possible origin for their abundance anomalies, giving particular attention to the lithium abundance anomalies. In particular, we investigate the possible physical mechanisms that could account for the simultaneous lithium overabundances and strong carbon underabundances. In the following, we describe the homogeneous redetermination of atmospheric parameters and lithium abundances for the WGB stars known to date in Sects. 2 and 3. We then place the 
A. Palacios et al.: Weak G-band stars on the H-R diagram

Table 1. Basic data and atmospheric parameters of weak G-band stars derived from the HIPPARcos catalogue for HIP stars (Col. 2).

\begin{tabular}{|c|c|c|c|c|c|c|c|c|c|c|c|c|c|}
\hline HD No. & HIP No. & $m_{v}$ & $B-V$ & $\pi$ & $\sigma(\pi)$ & $\sigma(\%)$ & $\log \left(L / L_{\odot}\right)$ & $\sigma$ & $T_{\text {eff }}$ & $\sigma$ & $\log g$ & $\sigma$ & Ref. \\
\hline 18474 & 13965 & 5.47 & 0.869 & 6.390 & 0.350 & 5.4 & 2.192 & 0.088 & 5140 & 110 & 2.59 & 0.20 & $\mathrm{H}$ \\
\hline 18636 & 13865 & 7.63 & 0.897 & 3.480 & 0.570 & 16.3 & 1.864 & 0.184 & 5080 & 110 & 2.90 & 0.29 & $\mathrm{H}$ \\
\hline 21018 & 15807 & 6.37 & 0.851 & 2.680 & 0.740 & 27.6 & 2.628 & 0.28 & 5300 & 80 & 2.21 & 0.39 & $\mathrm{H}$ \\
\hline 26575 & 19509 & 6.43 & 1.070 & 3.800 & 0.390 & 10.2 & 2.332 & 0.128 & 4690 & 110 & 2.29 & 0.24 & $\mathrm{H}$ \\
\hline 28932 & 21250 & 7.94 & 0.996 & 1.940 & 0.730 & 37.6 & 2.276 & 0.368 & 4880 & 90 & 2.42 & 0.48 & $\mathrm{H}$ \\
\hline 31274 & 22620 & 7.13 & 0.958 & 3.730 & 0.380 & 10.1 & 2.072 & 0.128 & 5040 & 80 & 2.68 & 0.24 & $\mathrm{H}$ \\
\hline 31869 & - & 9.27 & 0.92 & - & - & - & - & - & - & - & - & - & - \\
\hline 36552 & - & 8.08 & 0.84 & - & - & - & - & - & 5250 & - & 1.5 & - & - \\
\hline 40402 & 28184 & 8.55 & 0.930 & 1.870 & 1.390 & 74.3 & 2.044 & 0.688 & 5010 & 120 & 2.70 & 0.80 & $\mathrm{H}$ \\
\hline 49960 & 32734 & 8.34 & 1.020 & 1.030 & 0.760 & 73.7 & 2.676 & 0.68 & 4830 & 80 & 2.00 & 0.79 & $\mathrm{H}$ \\
\hline 56438 & 35016 & 8.10 & 1.033 & 1.820 & 0.640 & 35.1 & 2.284 & 0.344 & 4790 & 110 & 2.38 & 0.45 & $\mathrm{H}$ \\
\hline 67728 & 39867 & 7.54 & 1.106 & 2.160 & 0.670 & 31.0 & 2.384 & 0.308 & 4660 & 90 & 2.23 & 0.42 & $\mathrm{H}$ \\
\hline 78146 & 44636 & 8.57 & 1.160 & 0.840 & 0.990 & 117.8 & 2.824 & 1.064 & 4530 & 90 & 1.74 & 1.17 & $\mathrm{H}$ \\
\hline 82595 & - & 8.20 & 1.00 & - & - & - & - & - & 4910 & - & 1.5 & - & - \\
\hline 91805 & 51816 & 6.09 & 0.940 & 2.870 & 0.430 & 14.9 & 2.736 & 0.172 & 5350 & 80 & 2.11 & 0.28 & $\mathrm{H}$ \\
\hline 94956 & 53561 & 8.45 & 0.960 & 2.580 & 0.790 & 30.6 & 1.816 & 0.308 & 4950 & 110 & 2.90 & 0.41 & $\mathrm{H}$ \\
\hline 102851 & 57724 & 8.78 & 1.053 & 1.400 & 1.220 & 87.1 & 2.244 & 0.796 & 4760 & 110 & 2.40 & 0.91 & $\mathrm{H}$ \\
\hline 119256 & 66975 & 7.29 & 1.123 & 4.160 & 1.110 & 26.6 & 2.000 & 0.272 & 4870 & 400 & 2.69 & 0.38 & $\mathrm{H}$ \\
\hline 120213 & 68009 & 5.95 & 1.411 & 4.070 & 0.330 & 8.1 & 2.644 & 0.112 & 4120 & 100 & 1.76 & 0.22 & A \\
\hline 120170 & - & 9.03 & 0.93 & - & - & - & - & - & 5265 & - & 2.70 & - & - \\
\hline 124721 & - & 9.48 & 0.93 & - & - & - & - & - & - & - & - & - & _- \\
\hline 146116 & 79604 & 7.68 & 1.070 & 1.160 & 0.710 & 61.2 & 2.856 & 0.572 & 4720 & 80 & 1.78 & 0.68 & $\mathrm{H}$ \\
\hline 165462 & 88671 & 6.32 & 1.014 & 4.650 & 0.480 & 10.3 & 2.172 & 0.128 & 4840 & 110 & 2.50 & 0.24 & $\mathrm{H}$ \\
\hline 165634 & 88839 & 4.55 & 0.938 & 9.620 & 0.260 & 2.7 & 2.276 & 0.064 & 5060 & 260 & 2.48 & 0.17 & $\mathrm{H}$ \\
\hline 166208 & 88788 & 5.00 & 0.913 & 7.960 & 0.620 & 7.7 & 2.200 & 0.108 & 5050 & 110 & 2.55 & 0.22 & $\mathrm{H}$ \\
\hline 188328 & 97922 & 7.02 & 0.584 & 12.110 & 1.670 & 13.7 & 0.932 & 0.16 & 6040 & 90 & 4.13 & 0.27 & $\mathrm{H}$ \\
\hline 204046 & 105901 & 9.00 & 1.037 & 1.390 & 1.270 & 91.3 & 2.160 & 0.836 & 4790 & 110 & 2.50 & 0.94 & $\mathrm{H}$ \\
\hline 207774 & 107885 & 8.92 & 0.969 & 2.320 & 1.300 & 56.0 & 1.720 & 0.528 & 4940 & 80 & 2.99 & 0.64 & $\mathrm{H}$ \\
\hline
\end{tabular}

Notes. Last column (H or A) means that the atmospheric parameters were derived according to the Houdashelt tabular data or the photometric calibration of Alonso respectively (see text).

References. $\mathrm{H}=$ Houdashelt (2000). A = Alonso et al. (1999).

WGB stars in the HR diagram and make a tentative classification in terms of evolutionary status and initial masses in Sect. 4. In Sects. 5 and 6 we finally explore possible scenarios to consistently account for the light element ( $\mathrm{Li}, \mathrm{C}, \mathrm{N}$ and $\mathrm{O}$ ) abundances found in WGB stars based on the results of dedicated stellar evolution models.

\section{Atmospheric parameters}

The most complete list of weak G-band stars known up to now is presented in Table 1. So far, 28 WGB stars have been identified in the literature. This represents a very small fraction $(<1 \%)$ of $\mathrm{K}$ giants and makes these objects belong to a very rare class. To study their position in the HR diagram we firstly determined their effective temperature $T_{\text {eff }}$ by using both $(B-V)$ and $(V-I)$ colours from the HIPPARCos catalogue (van Leeuwen 2007) and the improved colour-temperature relations for giants from Houdashelt et al. (2000) or Alonso et al. (1999). Colours of some stars are affected by a little interstellar reddening which we took into account for the determination of $T_{\text {eff }}$ and the bolometric correction $B C$ by using a least-squares approximation obtained by varying accordingly the colour excess $E(B-V)$ and $E(V-I)$. The error on $T_{\text {eff }}$ is estimated from our interpolation processing in the Houdashelt et al. (2000) tabular data. The bolometric magnitude $M_{\text {bol }}$ and gravity $\log (g)$ are given by

$$
\begin{aligned}
M_{\mathrm{bol}} & =V-3.1 E(B-V)+B C(V)+5 \log (\pi)+5 \\
\log (g) & =\log (M)+0.4 M_{\mathrm{bol}}+4 \log \left(T_{\mathrm{eff}}\right)-12.505,
\end{aligned}
$$

where $\pi$ is the HIPPARCos parallax (van Leeuwen 2007) and $M$ the mass of the star in solar units. The $V$ magnitude is also taken from the release of the HIPPARCos catalogue by van Leeuwen (2007). In the preceding equation, gravity $\log (g)$ is little dependent on the mass of the star in the range $2-4 M_{\odot}$. Owing to the location of the stars in the HR diagram (see Fig. 1), we assumed $M=3.5 M_{\odot}$ for the calculation of $\log (g)$ in Table 1 . The error on $\log (g)$ is caused essentially by the error on $T_{\text {eff }}$. The errors on the luminosities are computed using the quoted uncertainties in the parallaxes and magnitudes. They may be huge for certain WGB stars because they propagate from ill-determined parallaxes. Atmospheric parameters given in Table 1 excellently agree with those calculated recently by other authors (Takeda et al. 2008; Wu et al. 2011).

\section{Lithium abundance}

Lithium abundance for most of the weak G-band stars, rederived from the available strengths of Li resonance line at $6707.8 \AA$, are given in Table 2. The equivalent widths of Li line and metallicities were taken from the literature (Hartoog 1978; Lambert \& Sawyer 1984). Solar metallicity was considered for the giants whose metallicity information was not available in the literature. Local thermodynamic equilibrium (LTE) stellar model atmospheres computed by Kurucz (1994) ${ }^{3}$ with convection option on, and the revised radiative transfer code MOOG originally written by Sneden (1973) were used for the analysis. The welltested line list (Reddy et al. 2002) in the region of Li resonance line $6707.8 \AA$ was adopted. Hyperfine structure and the $g f$ values of ${ }^{7} \mathrm{Li}$ components of $\mathrm{Li}$ line at $6707.8 \AA$ were taken from

3 www. kurucz.harvard.edu 


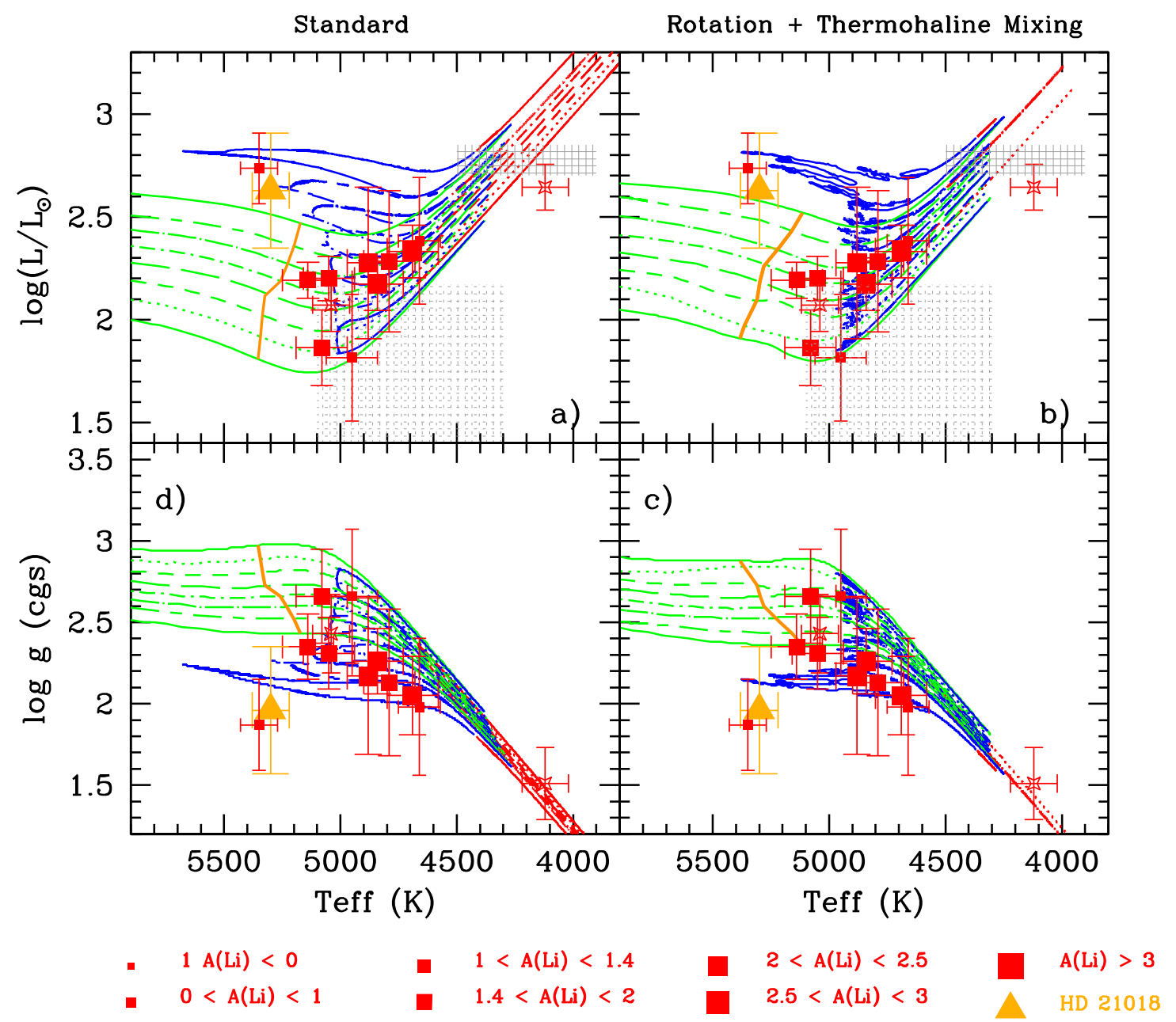

Fig. 1. Weak G-band stars with the best parallaxes (error on the $\log \left(L / L_{\odot}\right)$ lower than 0.5 dex) are plotted in the Hertzsprung-Russell diagram (upper panels) and in the ( $\left.\log g, T_{\text {eff }}\right)$ plane (lower panels) along with evolutionary tracks for models with initial masses $3,3.2,3.4,3.6,3.8,4.0$, 4.2 and $4.5 M_{\odot}$ as described in Sect. 4 (from bottom to top in the HR diagrams and in the reverse way, from top to bottom, in the (log $g$, $T_{\text {eff }}$ ) diagrams). Left panels represent classical models without rotation or diffusion processes, and right panels show models including rotation and thermohaline mixing. The bold line intersecting the racks on the left side of the plots marks the beginning of the first dredge-up. The size of the symbols is proportional to the Li content (the more lithium, the larger the symbol). Empty symbols are those for which no lithium abundance exists to date. The triangle in each of the plots represents star HD 21018, which is a spectroscopic binary. The shaded areas in the HR diagrams indicate the location of the Li-rich K giants, corresponding to the bump area for models with initial masses lower than $2.3 M_{\odot}$ and to the early-AGB phase for the models in the mass range presented here.

Hobbs et al. (1999). The NLTE corrections were applied using the recipe given by Lind et al. (2009).

The information on the lithium abundance in Table 2 is given for 19 stars. Lithium studies have not been attempted until now on those nine stars whose $\mathrm{Li}$ information is missing from Table 2. Among the 19 stars with available lithium, 11 giants can be qualified as "Li-rich". This denomination refers to an unexpectedly large $\mathrm{Li}$ abundance compared to the post dredge-up value of about 1.4 dex expected for giant stars at solar metallicity from standard stellar evolution theory (see this work and Brown et al. 1989). This represents about $39 \%$ of the WGB stars and almost $58 \%$ of the WGB whose lithium content has been investigated. The remaining eight stars are lithiumpoor. The WGB stars present a wide range of $\mathrm{Li}$ abundances from -0.2 to 3.0 dex. In contrast to $\mathrm{K}$ giants, $\mathrm{Li}$ overabundances are much more common in this subclass of stars, amounting to about $58 \%$ for those stars whose lithium region around $6707.8 \AA$ has been observed. No weak G-band star is found to be more $\mathrm{Li}$ abundant than the main-sequence or interstellar medium value
$(A(\mathrm{Li}) \sim 3.28)$. This very high percentage by itself adds to the peculiarity of WGB stars among other types of lithium-rich stars, in particular Li-rich giants which are very rare.

We will discuss the possible origin of these unexpectedly large lithium abundances in greater detail in Sect. 6.

\section{HR diagram}

To better evaluate the evolutionary status of the weak G-band stars (also with respect to that of the Li-rich $\mathrm{K}$ giants), we computed two grids of dedicated models for stars with solar metallicity (the solar chemical composition adopted as a reference is that of Grevesse \& Noels 1993) in the mass range 3 to $4.5 M_{\odot}$. The models were all evolved from the pre-main sequence to the early AGB phase. They were computed using the STAREVOL V3.0 code, which has been extensively described in Siess (2006), Palacios et al. (2006), and Decressin et al. (2009). Standard models displayed in panels a) and c) of Fig. 1 do not include mixing outside the convective regions, and the convective boundaries are fixed according to the Schwarzschild criterion 
Table 2. Chemical abundances and rotational velocities of weak G-band stars.

\begin{tabular}{lcccccccccc}
\hline \hline $\mathrm{HD}$ & {$[\mathrm{Fe} / \mathrm{H}]$} & $A(\mathrm{Li})$ & $W_{\lambda}$ & $A(\mathrm{Li})$ & $A(\mathrm{Li})_{\mathrm{NLTE}}$ & {$[\mathrm{C} / \mathrm{H}]^{b}$} & {$[\mathrm{~N} / \mathrm{H}]^{b}$} & {$[\mathrm{O} / \mathrm{H}]$} & ${ }^{12} \mathrm{C} /{ }^{13} \mathrm{C}$ & $V \sin i$ \\
\hline 18474 & $-0.10^{a}$ & $1.42^{a}$ & $29^{a}$ & 1.33 & 1.48 & -1.6 & 0.2 & - & - & $1.9^{g}$ \\
18636 & $-0.11^{b}$ & $1.77^{b}$ & $66^{b}$ & 1.71 & 1.853 & - & - & - & - & - \\
$21018^{p}$ & - & $2.89^{c}$ & $245^{c}$ & 3.13 & 3.06 & - & - & - & - & $20.1^{g}$ \\
26575 & $-0.01^{b}$ & $2.02^{b}$ & $239^{b}$ & 2.16 & 2.34 & - & - & - & - & - \\
28932 & - & $2.25^{a}$ & $158^{a}$ & 2.04 & 2.212 & - & - & - & - & - \\
31274 & $-0.27^{b}$ & $<0.52^{b}$ & $<4^{b}$ & -0.67 & - & - & - & - & - & \\
31869 & - & - & - & - & - & - & - & - & - & - \\
36552 & $-0.17^{b}$ & $2.58^{b}$ & $173^{b}$ & 2.64 & 2.70 & - & - & - & - & - \\
40402 & $-0.45^{b}$ & $2.44^{b}$ & $215^{b}$ & 2.60 & 2.586 & - & - & - & - & - \\
49960 & - & - & - & - & - & - & - & - & - & - \\
56438 & $-0.38^{b}$ & $1.43^{b}$ & $104^{b}$ & 1.59 & 1.774 & - & - & - & - & - \\
67728 & - & $0.76^{a}$ & $15^{a}$ & -0.22 & 0.06 & - & - & - & - & - \\
78146 & $-0.31^{b}$ & $1.01^{b}$ & $22^{b}$ & -0.27 & 0.01 & - & - & - & - & - \\
82595 & $-0.10^{b}$ & $1.14^{b}$ & $22^{b}$ & 0.89 & 1.136 & - & - & - & - & - \\
91805 & $0.0^{e}$ & $0.25^{b}$ & $03^{b}$ & 0.06 & 0.202 & -1.4 & 0.6 & $0.1^{e}$ & - & - \\
94956 & - & $1.07^{a}$ & $15^{a}$ & 0.65 & 0.834 & - & - & - & - & - \\
102851 & - & - & - & - & - & - & - & - & - & - \\
119256 & - & - & - & - & - & - & - & - & - & - \\
120213 & - & - & - & - & - & - & - & - & - & - \\
120170 & $-0.07^{a}$ & $3.03^{a}$ & $253^{a}$ & 3.14 & 3.02 & - & - & - & - & - \\
124721 & - & - & - & - & - & - & - & - & - & - \\
146116 & - & - & - & - & - & - & - & - & - & - \\
165462 & - & $2.05^{a}$ & $141^{a}$ & 1.87 & 2.068 & - & - & - & - & - \\
$165634^{p}$ & $-0.15^{f}$ & $0.75^{f}$ & - & - & - & -1.49 & 0.23 & $-0.38^{e}$ & $4.1^{e}$ & $1.49^{h}$ \\
$166208^{p}$ & $+0.2^{a}$ & $1.65^{a}$ & $74^{a}$ & 1.73 & 1.90 & -0.9 & 0.8 & - & $4.5^{d}$ & $2.9^{g}$ \\
188328 & - & - & - & - & - & - & - & - & - & $6.3^{g}$ \\
204046 & - & - & - & - & - & - & - & - & - & - \\
207774 & - & $1.5^{a}$ & $30^{a}$ & 1.06 & 1.24 & - & - & - & - & - \\
\hline
\end{tabular}

References. ${ }^{(a)}$ Hartoog (1978), ${ }^{(b)}$ Lambert \& Sawyer (1984), ${ }^{(c)}$ From our spectra, ${ }^{(d)}$ Day (1980), ${ }^{(e)}$ Cottrell \& Norris (1978), ${ }^{(f)}$ Sneden et al. (1978), ${ }^{(g)}$ Glebocki \& Gnacinski (2005), ${ }^{(h)}$ Hekker \& Meléndez (2007), ${ }^{(p)}$ binary.

for the convective instability. No overshooting is included. The tracks associated with the rotating models are displayed in panels b) and d) of the same figure. They were computed assuming solid-body rotation in the convective envelopes (which should result in a minimum estimate of the amount of shear mixing that possibly develops in the radiative region and connects the convective envelope and the nuclear active regions; see Palacios et al. 2006), and rotation and thermohaline mixing were included from the Zero Age Main- Sequence (e.g. ZAMS) up to the earlyAGB phase. The formalism used is the same as in Charbonnel \& Lagarde (2010) and the reader is referred to this paper for more details. We briefly recall that we assume an efficient thermohaline mixing following Ulrich (1972), which might turn out to be a maximum efficiency approach given the lack of actual good description of this thermodynamic instability in the astrophysical regime (Cantiello \& Langer 2010). As in Charbonnel \& Lagarde (2010), the rotation velocity of the models when they reach the main sequence is assumed to correspond to $45 \%$ of their critical velocity, which results in mean equatorial rotation velocities of about $200 \mathrm{~km} \mathrm{~s}^{-1}$. Mass loss was included in all models using the classical Reimers law (Reimers 1975) up to the early AGB phase.

Figure 1 displays the luminosity and effective gravity of the WGB stars as a function of the effective temperature together with the evolutionary tracks. Only objects with sufficiently accurate parallaxes (e.g. $\left.\epsilon\left(\log \left(L / L_{\odot}\right)\right)<0.5\right)$ are shown. The shaded areas in panels a) and b) indicate the location in the HR diagram of the Li-rich K giants as reported in Kumar et al. (2011) and Charbonnel \& Balachandran (2000). Only a small overlap exists and Li-rich WGB stars that are more luminous appear to be clearly detached from the bulk of Li-rich K giants. The thick line intersecting the tracks on the left part of the plots indicates the beginning of the first dredge-up. Clearly, WGB stars appear to be stars in the mass range 3 to $4.5 M_{\odot}$ at the clump. Their position in the HR diagram makes determining their evolutionary status ambiguous: they could either be on the red giant branch, undergoing the first dredge-up, or be in the core helium burning phase. Cottrell \& Norris (1978) already suggested that WGB stars could be core helium burning stars in their study of HD 91805, but in contrast to what they thought, WGB stars are not past the helium flash because this event only occurs in stars with a mass lower than approximately $2.3 M_{\odot}$ (see e.g. Palmerini et al. 2011). Cottrell \& Norris also suggested that meridional circulation induced by rotation in main-sequence stars of intermediate mass could be a possible mechanism to explain the strong depletion of carbon in WGB stars. We therefore computed models including this transport process along with turbulent shear and thermohaline mixing to test this hypothesis.

Rotation velocities $(v \sin i)$ were measured for a limited number of WGB stars and are reported in Table 2. Figure 2 shows the evolution of the surface equatorial velocity of our rotating models during the RGB and subsequent evolutionary phases. To mimic a mean $v \sin i$ associated to what can be considered a mean equatorial velocity given by $1 \mathrm{D}$ stellar evolution models, we multiplied the velocity given by our models by $\frac{\pi}{4}$, following Chandrasekhar \& Münch (1950). The observational data seem to suggest rotation rates lower (by a factor of two approximately) than what is predicted by our models. Conservatively adopting a solid-body rotation law for the convective envelope favours higher surface rotation rates. Using a 


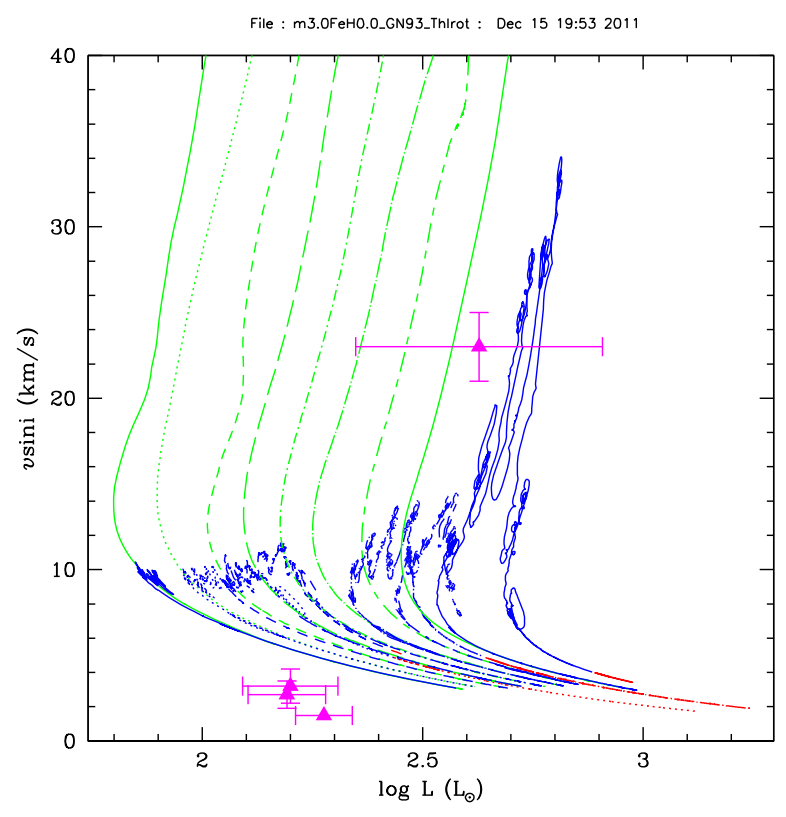

Fig. 2. $v \sin i$ as a function of luminosity for the rotating models with initial masses from 3.0 to $4.5 M_{\odot}$ (from left to right). The triangles are the observational values for HD 18474, HD 21018, HD 166208, HD 188328, and HD 165634 as listed in Table 2.

probably more realistic differential rotation in the extended convective envelopes of these stars as they evolve through the giant phases will lead to lower surface rotation velocities (see Brun \& Palacios 2009). HD 21018 is quite well reproduced, on the other hand, considering the large error bars on the parallax. The very slow velocities estimated for the five stars quoted in Table 2 are consistent with what could be expected from stars undergoing core helium burning.

As can be seen from Fig. 1, the evolutionary tracks are slightly modified by the action of rotation and thermohaline mixing, having less extended and noisier blue loops. The tracks nonetheless match the region occupied by the WGB stars as well as the standard models do.

Below, we compare the models, standard and with rotation, to the abundance data and discuss the possible origin of the WGB stars in the light of this comparison.

\section{Abundances}

The position of WGB stars in Fig. 1 indicates that they are bright stars of intermediate mass. The expected evolution of the surface abundances of lithium, carbon, nitrogen, and carbon isotopic ratio for our standard and rotating models is shown in Fig. 3, together with the values reported in Table 2 for the WGB stars. In this figure, the squares in different shades of blue/violet correspond to HD 166208 (violet), HD 165634 (brown), HD 91805 (cyan), and HD 18474 (blue).

\section{Lithium}

The evolution of the surface abundance of lithium in standard (left) and rotating models (right) is displayed in the first row. The weak G-band stars are either Li-rich or Li-depleted.

For those stars that have Li abundances lower than 1.4 dex, we clearly see that standard stellar evolution models fail to reproduce them, the level reached after the first and second dredge-up episodes being of about 1.4 dex. On the contrary, the models including rotation-induced mixing (e.g. meridional circulation + secular turbulent shear instability) and thermohaline mixing undergo lithium depletion already during the main-sequence phase and achieve $A(\mathrm{Li}) \approx 0$, which agrees very well with the NLTE corrected lithium abundance derived for HD 67728, HD 78146, and HD 91805.

The remaining WGB stars exhibit lithium abundances that would be typical of first dredge-up dilution in standard (nonrotating) models and in models with slow rotation on the ZAMS $\left(50 \mathrm{~km} \mathrm{~s}^{-1}\right)$. This can be seen from the upper panels of Fig. 3, where the grey shaded area corresponds to the location of tracks for slow rotating models. Among those for which lithium lines were actually observed, the proportion of WGB stars with $A(\mathrm{Li})>1.4$ dex is about $58 \%$, which means either that a large proportion of core helium burning WGB stars are Li-rich, or that a large fraction of the WGB stars are young red giants in which the first dredge-up is not yet completed.

We postpone a more detailed discussion to Sect. 6 .

\section{Beryllium}

The evolution of the surface Be abundance in our models leads to a mean post-dredge-up value (first and second) of about -0.2 dex for the standard models, while the models including rotation and thermohaline mixing reach $A(\mathrm{Be}) \approx-0.65 \mathrm{dex}$. These values are to be compared with the abundances estimated from IUE spectra by Parthasarathy et al. (1984) of about -0.5 dex. Errorbars on observed values are not detailed in the original paper but the value is considered an upper limit so that non-standard models are favoured again here.

\section{Carbon}

Actual derivation of carbon abundances exist only for some stars with known parallaxes, which are represented together with our model predictions in the second row of Fig. 3. While models including rotation-induced and thermohaline mixing lead to $[\mathrm{C} / \mathrm{H}] \approx-0.25$ dex by the end of the core helium burning phase, a depletion higher by about 0.05 dex than predicted for classical stellar evolution models, these values are far from reaching the extreme underabundances derived for HD 166208, HD 165634 , HD 91805, and HD 18474 (see also Table 2).

The combined action of meridional circulation and turbulent shear and thermohaline mixing that has been proposed as a physical process to explain light-elements ( $\mathrm{Li}, \mathrm{C}$ and N) abundance anomalies in RGB stars (Charbonnel \& Lagarde 2010) clearly fails here to account for the very high carbon depletion in WGB stars when using the same set of parameters as those adopted for lower mass stars. Among the WGB stars for which $[\mathrm{C} / \mathrm{H}]$ is available, HD 91805 is "normal" in terms of lithium (and also nitrogen, see below) abundance when compared to non-standard models that include rotation and thermohaline mixing, while the other three stars are Li-rich $(A(\mathrm{Li}) \geq$ 1.4 dex).

The carbon deficiency, the common feature of WGB stars, therefore seems to be decorrelated from the other abundance anomalies, in particular the lithium content, and could have a different origin.

\section{Nitrogen}

Nitrogen abundances are available from the literature for the same stars for which the carbon abundance was derived. 


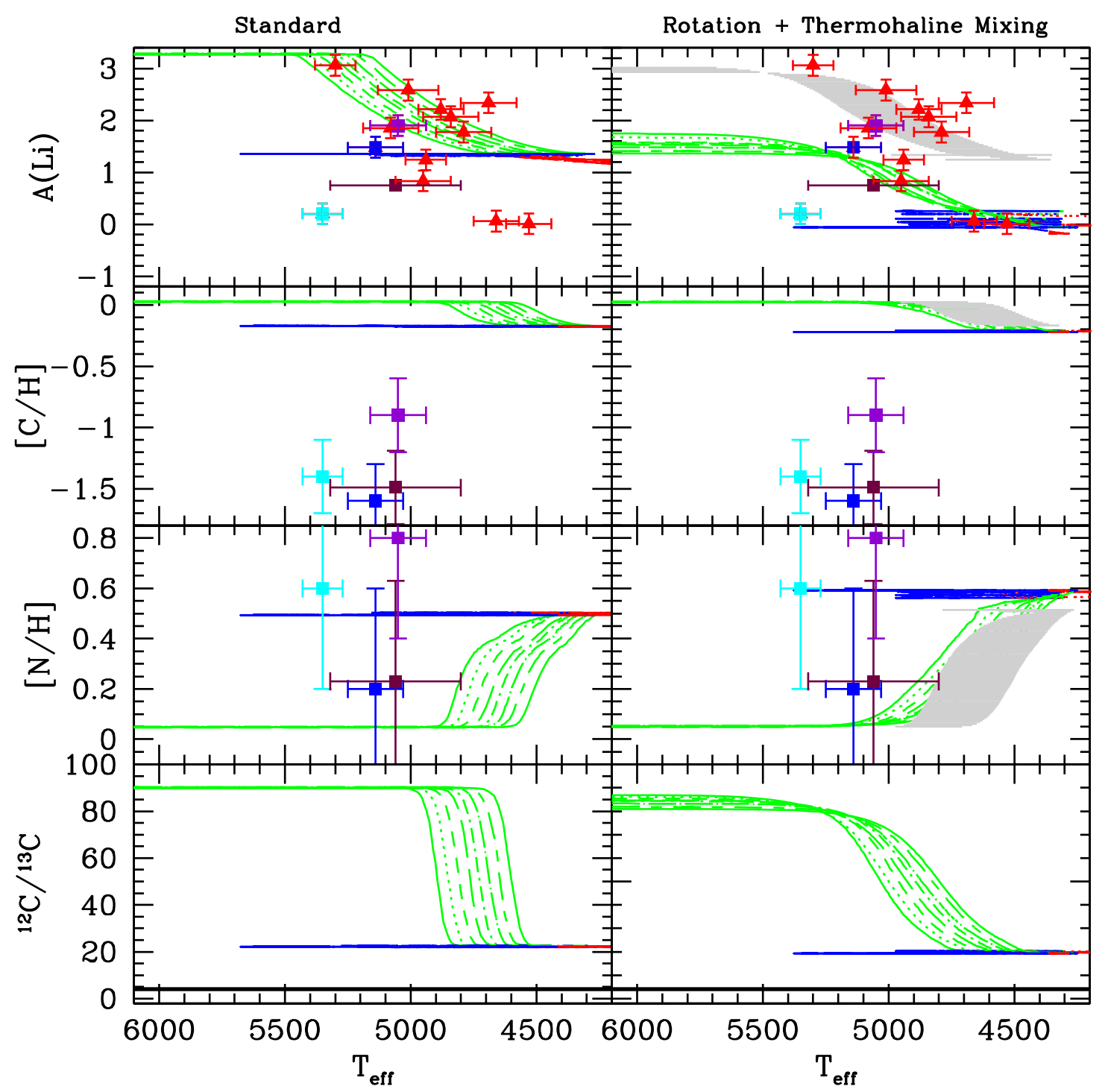

Fig. 3. Abundances of lithium, carbon, nitrogen, and the carbon isotopic ratio as a function of the effective temperature for the objects listed in Table 2. A conservative error bar of 0.2 dex was adopted for the NLTE lithium shown in the upper panels. The tracks are for standard (left column) and rotating models (right column) with masses ranging from 3.0 to $4.5 M_{\odot}$ (from left to right). The colours of the tracks indicate the evolutionary phase: black for main sequence, green for RGB, blue for central He burning and red for AGB phase. Squares in different shades of blue are used in the upper panel to represent those stars for which $[\mathrm{C} / \mathrm{H}]$ and $[\mathrm{N} / \mathrm{H}]$ is also available from the literature (see text). In the lower panels, the thick black line at ${ }^{12} \mathrm{C} /{ }^{13} \mathrm{C}=4$ represents the carbon isotopic ratio found in HD 16634 (Sneden et al. 1978) and in HD 166208 (Day 1980). The grey areas in the left column plots show the region occupied by tracks for rotating models with $v_{\text {ZAMS }}=50 \mathrm{~km} \mathrm{~s}^{-1}$ (see text).

The third row (from the top) of Fig. 3 displays the evolution of the $[\mathrm{N} / \mathrm{H}]$ as predicted by stellar evolution and the abundances derived for HD 166208, HD 165634, HD 91805, and HD 18474. In our non-standard models, nitrogen is enhanced during the RGB phase (rising parts of the tracks) as a result of the continuously ongoing rotation-induced mixing that allows bridging the gap between the convective envelope and the hydrogen burning shell. Thermohaline mixing only develops near the core, but anyway remains much slower than meridional circulation and shear-induced turbulence, and is not the lead process shaping the surface abundance pattern of our models.

Both standard and non-standard models lead to surface abundances at the core helium burning phase that agree with the observational data within the (large) error bars. However, we can distinguish two groups:

1. the more nitrogen-rich stars HD 91805 (cyan square at $T_{\text {eff }}=$ $5350 \mathrm{~K}$ ) and HD 166208 (purple square at $T_{\text {eff }}=5050 \mathrm{~K}$ ) are clearly better fitted by rotating models that undergo core helium burning, in particular HD 91805;

2. the nitrogen abundances of HD 165634 and HD 18474 fall in between $[\mathrm{N} / \mathrm{H}]_{\mathrm{SGB}}(\approx 0.05 \mathrm{dex})$ and $[\mathrm{N} / \mathrm{H}]_{\text {clump }}(\approx 0.6 \mathrm{dex})$ as predicted by both our sets of models. This is quite puzzling and definitely does not help to pinpoint the evolutionary status of these stars.

\section{Origin of the peculiar chemical pattern of WGB stars}

From the preceding sections Sects. 4 and 5, weak G-band stars appear to be intermediate-mass stars with initial masses in the range 3 to $4.5 M_{\odot}$, which are undergoing either the first dredgeup or are in the core helium burning stage. A large fraction of these stars is lithium-rich $(A(\mathrm{Li})>1.4 \mathrm{dex})$, and all of them present large carbon underabundances even when compared to 
non-standard stellar evolution models that include thermohaline mixing and rotation.

When trying to understand the observations, one needs to be able to account for the following features:

1. all WGB stars are carbon-deficient, and for the four stars with available measurements for the carbon abundance, $[\mathrm{C} / \mathrm{Fe}]<-1$ dex; carbon isotopic ratio is also seemingly very low (around equilibrium value 4 ). The observed underabundance is larger by at least 1 dex than that predicted by standard and non-standard models;

2. a large fraction (about 58\%) of the WGB stars for which lithium lines were observed exhibit $A(\mathrm{Li})>1.4 \mathrm{dex}$;

3. lithium and nitrogen surface abundances as well as evolutionary status are well-matched by stellar evolution models that include rotation and thermohaline mixing for those stars that present a low lithium surface abundance;

4. the rotation velocity of WGB stars with measured $v \sin i$ is marginally reproduced by rotating stellar evolution models with $v_{\text {ZAMS }} \approx 200 \mathrm{~km} \mathrm{~s}^{-1}$.

\section{1. "Li-poor" weak G-band stars}

As partly discussed in Sect. 5, lithium-poor WGB stars are actually normal except for their carbon abundance. HD 91805 is used as a prototype because we have access to its luminosity, temperature, gravity, lithium, carbon, and nitrogen abundances. This star is well-fitted by models that include rotation, rotationinduced mixing (meridional circulation + shear turbulence), and thermohaline mixing with an initial mass of about $4.5 M_{\odot}$.

If we consider an internal origin for the carbon underabundance, as proposed by Cottrell \& Norris (1978) and Tomkin et al. (1984), the evolutionary status of RGB stars should be ruled out. Considering their mass, WGB stars experience the end of the first dredge-up while already undergoing core helium burning, and no such extra mixing processes as rotation-mixing or thermohaline mixing are expected (and actually seen in the models) to be sufficiently efficient to create the high carbon depletion. Furthermore, admitting that some extra-mixing acts as an overshooting and increases the depth of the dredge-up, the very large extent of the convective envelope at this stage implies a very high dilution factor that will prevent the carbon abundance and carbon isotopic ratio from dropping to the observed value within the duration of the RGB phase. Even though the mixing reaches down to the region where $\mathrm{CN}$-cycle occurs, this region is far too shallow compared to the convective envelope for this late region to be completely processed.

Meridional circulation and shear turbulence are actually efficient in earlier evolutionary phases, as can be seen in Fig. 3, but they mainly act on the lithium abundance and the associated mixing does not reach the carbon-depleted regions that are located deeper in the core. Contrary to what was suggested by Lambert \& Sawyer (1984) or Cottrell \& Norris (1978), it seems very unlikely that carbon depletion is the result of internal mixing during the early evolution of the progenitors of WGB stars.

During core helium burning, on the other hand, the HBS crosses the composition gradient left by the dredge-up when the stars are located at the "turning point" of the blue loop, and then the regions were $\mathrm{CN}$-cycling occurs are again accessible to extra-mixing processes. However, the physical distance separating the nucleosynthetic regions from the now much shallower convective envelope is very long (more than $22 R_{\odot}$ ): for an extramixing process to be efficient its characteristic timescale would need to be shorter than the evolutionary timescale of about 20 to $200 \mathrm{Myr}$ that is typical for stars in the mass range considered here. In terms of diffusion coefficient, this would imply $D_{\text {extramix }} \approx 10^{8}-10^{10} \mathrm{~cm}^{2} \mathrm{~s}^{-1}$. The total diffusion coefficient obtained in our non-standard models $\left(D_{\mathrm{MC}}+D_{\text {turb }}+D_{\mathrm{Thl}}\right)$ is always lower by at least one order of magnitude. These high rates of mixing are difficult to obtain using the available prescriptions for hydrodynamical instabilities.

Altogether these points indicate that the extra-mixing processes included so far in our stellar evolution models (meridional circulation + turbulent shear + thermohaline mixing) cannot produce the huge carbon underabundances, but do reproduce the lithium and nitrogen abundances measured for three of the WGB stars quite well if likely rotation velocities are adopted for their main-sequence progenitors.

\subsection{Li-rich weak $G$ band stars}

As already mentioned several times, WGB stars with $A(\mathrm{Li}) \geq$ 1.4 dex represent a high percentage of these stars. These lithium abundances will be considered an evidence for lithium overabundances if the stars are evolved past the first dredge-up, but would be within the expected range for stars that are currently undergoing the first dredge-up.

We analysed the available observational data using both interpretations and the consequences of adopting one or the other point of view for the origin of the peculiar abundance patterns of WGB stars.

\section{WGB stars as core helium burning stars}

If one assumes from their location at the clump in the HR diagram and the discussion in the previous paragraph concerning the evolutionary status of the Li-poor ones that WGB stars are core helium burning stars, then lithium enrichment is everything but an exceptional feature among this stellar subclass. The Lirich stars are rare among other classes of giants: in the case of low-mass stars, they are the result of seemingly very short enrichment episodes at which we manage to catch a glimpse for only $1-5 \%$ of the RGB stars (Charbonnel \& Balachandran 2000; Palacios et al. 2001). For more massive stars, they are produced by Li-enrichment episodes in stars with $M_{\star} \geq 5 M_{\odot}$ that undergo Hot Bottom Burning (e.g. HBB) during the thermal pulse AGB phase (Palmerini et al. 2011).

Weak G-band stars do not appear to be on the TP-AGB phase (too warm and not luminous enough). During the core helium burning phase there is no such thing as HBB, and to explain Lienrichment by internal mixing, one should invoke Cool Bottom Processing (e.g. CBP), a general denomination for any unspecified mechanism able to connect the convective envelope with deeper regions where nuclear reactions occur.

CBP in relation with rotation-induced hydrodynamical instabilities has been proposed to account for Li enrichment at the RGB bump (Palacios et al. 2001) in the lithium flash scenario. In this scenario, the convective envelope of the RGB star is enriched with lithium when the outer regions of the HBS where ${ }^{7} \mathrm{Li}(\mathrm{p}, \alpha){ }^{4} \mathrm{He}$ occurs, become convectively unstable and connect with the envelope. This episode is very prompt in time, and is rapidly followed by a deepening of the extra-mixing. Carbon and lithium abundances subsequently decrease at the surface.

While one could be tempted to transpose this scenario to the Li-rich WGB stars, a difficulty arises because it excludes a simultaneous increase of $\mathrm{Li}$ and depletion of carbon. This could be overcome if we consider that the stars already present 
a strong carbon depletion prior to the red giant phase. Another difficulty resides in the timescales: short-time enrichment is difficult to reconcile with the fact that almost $40 \%$ of the stars in the WGB class are Li-rich, but keeping in mind that WGB stars are very rare per se, this difficulty might not be as difficult to overcome as it may appear at first sight.

Lambert \& Sawyer (1984) suggested that the abundance anomalies of WGB stars, including Li enrichment and strong carbon depletion, could be caused by diffusion in the early stages of the evolution of A stars. They speculated that WGB stars could be the progeny of Ap stars, which are well-known magnetic Chemical Peculiar stars. The role they attributed to magnetism is not at all developed, because they only use the fact that Ap stars are rare and that the paucity of WGB stars is consistent with such a filiation. Considering the masses derived for the WGB stars in Sect. 4, they cannot be descendants of A stars and are the progeny of late-B type stars. If we still consider the possibility that WGB stars are descendants of magnetic Bp stars, we can expect effects in these stars that are similar to those in magnetic Ap stars and a similar evolution. Concerning a diffusion scenario including the combination of gravitational settling and radiative accelerations, any abundance stratification that would be built during the main sequence will be erased by the first dredge-up (Richard, priv. comm.). Moreover, for stars with $T_{\text {eff }}>12000 \mathrm{~K}$ on the main sequence, the effects of diffusion are expected to be essentially confined to the atmosphere, and non-magnetic evolution models by Turcotte \& Richard (2003) show that no specific accumulation or depletion of carbon is expected in the interior of these stars that could appear at their surface when they will evolve up the giant branch.

The evolutionary models for Ap/Bp stars are not yet available since the effects of magnetic fields and how to take them into account is still a matter of debate. Still, Charbonnel \& Zahn (2007) suggested that magnetic Ap stars give rise to the very few RGB stars that present light elements abundances $\left({ }^{3} \mathrm{He},{ }^{7} \mathrm{Li}\right.$, $\mathrm{C}, \mathrm{N})$ that comply to the predictions of standard and rotating models. Strong magnetic fields are indeed invoked as a means to inhibit the thermohaline mixing that is thought to be responsible for the light elements abundance variations seen at the surface of RGB stars that have evolved past the bump. Following this work and considering that for intermediate-mass stars, thermohaline mixing is inefficient unless maybe during the TP-AGB phase, which is at a more advanced evolutionary stage than that attributed to WGB stars, magnetic fields are not expected at all to favour carbon depletion of any sort, and the scenario proposed by Lambert \& Sawyer (1984) is discarded.

Mass transfer (or mass accretion) appears as one of the very few options left to try and understand the WGB phenomenon. From the HST UV spectra Böhm-Vitense et al. (2000) found evidence for the presence of a white dwarf companion to the weak G-band star HD 165634. On the other hand, Tomkin et al. (1984) searched for binarity in a sample of seven WGB stars of the northern hemisphere, finding a binarity rate of $15-40 \%$, which is not very different from that expected for normal $\mathrm{K}$ giants. However, this result is questionable since the number of targets selected was quite small and these observers did not exhibit the persistence that is really needed for an investigation of radial velocity variability because they stopped their observations after only two years (see arguments also given by Griffin 1992).

For the binary scenario to work, the secondary of the system should also be a star from which the accreted matter would be carbon-depleted (and possibly sometimes also lithium enriched). According to Forestini \& Charbonnel (1997, see their Table 8), stars in the mass range 5-6 $M_{\odot}$ at solar metallicity are expected to produce yields that show such a chemical imprint. In their models, they predict that the yields of ${ }^{7} \mathrm{Li}$ are positive because of the operation of efficient HBB, and more interestingly, that the net yields of ${ }^{12} \mathrm{C}$ are negative with values up to $-1.21 \times 10^{-2} M_{\odot}$. Forestini \& Charbonnel (1997) do not give any expected decrease of $\mathrm{C}$ at the surface of the secondary. They find lifetimes of $111 \mathrm{Myr}$ and $65.8 \mathrm{Myr}$ for their $5 M_{\odot}$ and $6 M_{\odot}$, respectively, at solar metallicity, to be compared with the $473 \mathrm{Myr}$ and $210 \mathrm{Myr}$ for their $3 M_{\odot}$ and $4 M_{\odot}$, respectively. These numbers imply that the mass accretion from the more massive shorter lived primary onto the secondary would occur during the early main-sequence evolution of the latter. Because main-sequence stars in the mass range 3 to $4.5 M_{\odot}$ have a radiative envelope, it is likely that the material that would be accreted would remain at the surface and possibly slowly diffuse inwards. If WGB stars are intermediatemass stars undergoing core helium burning, they have already evolved through the first dredge-up, one of the main signatures of which is the decrease of surface abundances of carbon and lithium, and the increase of nitrogen abundance. The dredge-up of carbon depleted, lithium-enhanced material could exacerbate the carbon depletion at the end of the dredge-up (but this need to be computed and checked before drawing any conclusion) but will certainly not affect the lithium abundance decrease since the point is that the base of the convective envelope enters regions where lithium is destroyed by proton captures.

Another uncertainty of the binary scenario is that of the yields of intermediate-mass stars. They indeed vary greatly from one source to another (see for instance Karakas \& Lattanzio 2007), making any conclusion elusive.

\section{WGB stars as young red giant stars (SGB/RGB)}

In contrast to the Li-poor WGB stars, that are very probably rotating core He burning stars with anomalous carbon depletion, it is much more difficult to really assess the evolutionary status of those that exhibit a lithium abundance larger than that expected after the completion of the first dredge-up.

Indeed, if no postulate is made concerning a common evolutionary status for all stars populating the weak G-band subclass, then a strong ambiguity arises. From the sole position in the HR diagram and the comparison with the stellar evolution predictions for lithium abundance (and marginally nitrogen), we cannot rule out the possibility that the so-called " $\mathrm{Li}$-rich" WGB stars could turn out to be just "normal" stars as far as lithium is concerned, meaning with lithium abundances consistent with models predictions, according to their evolutionary status and their rotational history.

Indeed, from Fig. 3 we see that the stars with large lithium abundance are well fitted by the standard tracks of intermediate mass stars that are undergoing the first dredge-up. According to these tracks, they should therefore not be considered as lithiumrich because the first dredge-up episode is not completed.

We may argue that the WGB stars should have experienced some rotational mixing because they are the progeny of B-type stars. The initial rotation spread observed in main-sequence Btype stars in the mass range 2 to $4 M_{\odot}$ as shown by Huang et al. (2010) is indeed very large, and it might well be that WGB stars are also the descendants of slow rotating late-B type stars.

In order to investigate the effect of a slower rotation velocity of the ZAMS progenitors of the WGB stars on their subsequent evolution, we have computed three models (with initial masses of $3.0 M_{\odot}, 3.5 M_{\odot}$, and $4.5 M_{\odot}$ ) for which we adopted $v_{\text {ZAMS }}=50 \mathrm{~km} \mathrm{~s}^{-1}$. The domain covered by these slow rotators is represented by the shaded area in Fig. 3 . 
$v_{\text {ZAMS }}=50 \mathrm{~km} \mathrm{~s}^{-1}$ corresponds to a ratio $v_{\text {eq }} / v_{\text {crit }} \approx 0.1$. With a rotation this slow, the transport generated by meridional circulation and turbulent shear instability is not very efficient during the main sequence evolution so that lithium is little depleted at the surface of our models when they reach the turnoff. The deepening of the convective envelope during the first dredge-up leads to a decrease of the surface lithium and carbon abundances and to an increase of the nitrogen abundance similar to that obtained for standard stellar evolution.

Although it has been commonly assumed that WGB stars shared the same evolutionary status, our models along with the chemical constraints (from lithium and nitrogen) that are available for WGB stars could be telling a different story. This subclass could be populated with the progeny of slow to mild rotating $\left(v_{\text {eq }} / v_{\text {crit }} \leq 0.45\right) \mathrm{B}$-type stars that are either experiencing the first dredge-up or that are past this phase and are core helium burning stars.

In this context the large carbon underabundance associated to the G-band weakness observed in all these stars as well as the narrow mass range become the only common features of WGB stars.

As mentioned earlier, a binary scenario is not to be excluded, but would deserve an in-depth study before any conclusions are drawn.

\section{Conclusions}

We have made a tentative re-analysis of the data existing for the WGB stars, in particular concerning fundamental parameters and lithium abundances. We confirmed that WGB stars are within the mass range 3-4.5 $M_{\odot}$. However, the scarcity of abundance determinations of $\mathrm{Li}, \mathrm{N}$, and $\mathrm{C}$ and the fact that the WGB stars are located at the clump where stellar evolution tracks corresponding to the subgiant, red giant, and core helium burning phases are intertwined prevent us from assessing any sound evolutionary status to the WGB stars. Concerning the chemical peculiarities, we used dedicated stellar evolution models to test their endogenous origin, and we showed that for those WGB stars that are not Li-rich, lithium and nitrogen abundances can be reproduced by rotating models. On the other hand, the carbon deficiency of all WGB stars is difficult to reconcile with an internal origin and we propose that it is decorrelated from the other chemical peculiarities. The case of lithium is still under debate because it is not clear, owing to the degeneracy of the evolutionary status, whether the WGB stars with $A(\mathrm{Li}) \geq 1.4$ dex are indeed Li-rich (core helium burning stars) or normal (subgiant or RGB stars undergoing the first dredge-up).

Carbon depletion might be associated to an early mass transfer from a more massive (5-6 $M_{\odot}$ ) companion under specific hypotheses, but yields of intermediate mass stars in that mass range are very uncertain and no firm conclusion should be drawn. Because the rate of binarity among WGB stars is far from clear, and is at least similar to that in normal giants, a new search for duplicity would be helpful.

It is quite clear from this study that more data are needed to shed light on the weak G-band stars puzzle. Considering the work by Miglio (2011) on the use of asteroseismic data to lift the status degeneracy between giants and clump stars, applying such an analysis to the oscillation spectra of WGB stars could be a very significant step in clarifying the evolutionary status of WGB stars.

Deriving proper abundances of light elements for the WGB stars is also needed to advance in understanding these overlooked puzzling stars.
Acknowledgements. The authors thank the referee for very thoughtful comments that have helped improve and clarify the work presented here. M.P. is thankful to Prof. Shoken Miyama, Prof. Ramanath Cowsik and Prof. Yoichi Takeda for their kind support, encouragement and hospitality. A.P. thanks Dr. O. Richard and Dr. F. Martins for fruitful discussions on diffusion and modelling.

\section{Appendix A: Notes on individual objects}

\section{A.1. HD 21018}

It is a well-known visual binary with an orbital period of 287 days. It has the highest $\mathrm{Li}$ among all weak G-band stars. Charbonnel (2000) suggested that it is undergoing the first dredge-up dilution. Owing to the duplicity, the position of this object in the HR diagram may be uncertain.

\section{A.2. $H D 165634$}

It is reported to have a white dwarf binary companion from the IUE spectral studies by Böhm-Vitense et al. (2000). Li information is unavailable for this star.

\section{A.3. $H D 188328$}

It is a well-known visual binary; both components are separated by about $2^{\prime \prime}$ according to Douglass et al. (2000). The position of this object in the HR diagram is near the main-sequence turn-off. Owing to the visual binarity the position in the HR diagram may be uncertain. Li information is unavailable.

\section{A.4. $H D 120213$}

It may be a binary star. It is classified as a weak G-band star in Hartoog et al. (1977). Additional studies on this star classified it as a Ba star of class 0.5 ( $\mathrm{Lu} \mathrm{1991).} \mathrm{In} \mathrm{general,} \mathrm{most} \mathrm{of} \mathrm{the}$ $\mathrm{Ba}$ stars are suggested to be binaries.

\section{A.5. HD 166208}

HD 166208 is a spectroscopic binary with a period of 5.5 years, low amplitude $\left(3 \mathrm{~km} \mathrm{~s}^{-1}\right)$, and moderate eccentricity $(0.4)$ (Griffin 1992). Griffin (1992) emphasizes the point that the eccentricity of the orbit of HD 166208 and its small mass function make a possible transfer of material between the components during a giant-phase evolution of the companion distinctly less likely that in the cases of barium stars.

For a few stars, like HD 36552, HD 82595, and HD 120170 , luminosities are not derived because of the lack of HIPPARCos parallaxes. There are no basic data for HD 124721 and HD 31869 available in the literature.

\section{References}

Alonso, A., Arribas, S., \& Martínez-Roger, C. 1999, A\&AS, 140, 261 Bidelman, W. P. 1951, ApJ, 113, 304

Böhm-Vitense, E., Carpenter, K., Robinson, R., Ake, T., \& Brown, J. 2000, ApJ, 533, 969

Brown, J. A., Sneden, C., Lambert, D. L., \& Dutchover, Jr., E. 1989, ApJS, 71, 293

Brun, A. S., \& Palacios, A. 2009, ApJ, 702, 1078

Cameron, A. G. W., \& Fowler, W. A. 1971, ApJ, 164, 111

Cantiello, M., \& Langer, N. 2010, A\&A, 521, A9

Chandrasekhar, S., \& Münch, G. 1950, ApJ, 111, 142

Charbonnel, C., \& Balachandran, S. C. 2000, A\&A, 359, 563

Charbonnel, C., \& Do Nascimento, Jr., J. D. 1998, A\&A, 336, 915

Charbonnel, C., \& Lagarde, N. 2010, A\&A, 522, A10

Charbonnel, C., \& Zahn, J.-P. 2007, A\&A, 476, L29 
Cottrell, P. L., \& Norris, J. 1978, ApJ, 221, 893

Day, R. W. 1980, Ph.D. Thesis, Texas Univ., Austin

Decressin, T., Mathis, S., Palacios, A., et al. 2009, A\&A, 495, 271

Douglass, G. G., Mason, B. D., Rafferty, T. J., Holdenried, E. R., \& Germain, M. E. 2000, AJ, 119, 3071

Drake, J. J., \& Lambert, D. L. 1994, ApJ, 435, 797

Forestini, M., \& Charbonnel, C. 1997, A\&AS, 123, 241

Glebocki, R., \& Gnacinski, P. 2005, VizieR Online Data Catalog, 3244, 0

Gratton, R. G., Sneden, C., Carretta, E., \& Bragaglia, A. 2000, A\&A, 354, 169

Grevesse, N., \& Noels, A. 1993, in Origin and Evolution of the Elements, ed.

N. Prantzos, E. Vangioni-Flam, \& M. Casse, 15

Griffin, R. F. 1992, The Observatory, 112, 219

Hartoog, M. R. 1978, PASP, 90, 167

Hartoog, M. R., Persson, S. E., \& Aaronson, M. 1977, PASP, 89, 660

Hekker, S., \& Meléndez, J. 2007, A\&A, 475, 1003

Hobbs, L. M., Thorburn, J. A., \& Rebull, L. M. 1999, ApJ, 523, 797

Houdashelt, M. L., Bell, R. A., \& Sweigart, A. V. 2000, AJ, 119, 1448

Huang, W., Gies, D. R., \& McSwain, M. V. 2010, ApJ, 722, 605

Jasniewicz, G., Parthasarathy, M., de Laverny, P., \& Thévenin, F. 1999, A\&A, 342,831

Karakas, A., \& Lattanzio, J. C. 2007, PASA, 24, 103

Kumar, Y. B., Reddy, B. E., \& Lambert, D. L. 2011, ApJ, 730, L12

Kurucz, R. 1994, Solar abundance model atmospheres for 0, 1, 2, 4, $8 \mathrm{~km} \mathrm{~s}^{-1}$, Kurucz CD-ROM No. 19 (Cambridge, Mass.: Smithsonian Astrophysical Observatory), 19

Lambert, D. L., \& Sawyer, S. R. 1984, ApJ, 283, 192

Lebzelter, T., Uttenthaler, S., Busso, M., Schultheis, M., \& Aringer, B. 2012, A\&A, 538, A36

Lind, K., Asplund, M., \& Barklem, P. S. 2009, A\&A, 503, 541
Lu, P. K. 1991, AJ, 101, 2229

Miglio, A. 2012, in Red Giants as Probes of the Structure and Evolution of the Milky Way, ed. A. Miglio et al. (Springer), in press [arXiv: 1108.4555]

Monaco, L., Villanova, S., Moni Bidin, C., et al. 2011, A\&A, 529, A90

Palacios, A., Charbonnel, C., \& Forestini, M. 2001, A\&A, 375, L9

Palacios, A., Charbonnel, C., Talon, S., \& Siess, L. 2006, A\&A, 453, 261

Palmerini, S., Cristallo, S., Busso, M., et al. 2011, ApJ, 741, 26

Parthasarathy, M., \& Jasniewicz, G. 2000, in 24th IAU GA, IAU Joint Discussion, 13, 38

Parthasarathy, M., \& Jasniewicz, G. 2002, in SF2A-2002: Semaine de

l'Astrophysique Française, ed. F. Combes, \& D. Barret, 555

Parthasarathy, M., \& Rao, N. K. 1980, Ap\&SS, 73, 251

Parthasarathy, M., Sneden, C., \& Bohm-Vitense, E. 1984, PASP, 96, 44

Rao, N. K. 1978, MNRAS, 185, 585

Reddy, B. E., Lambert, D. L., Hrivnak, B. J., \& Bakker, E. J. 2002, AJ, 123, 1993

Reimers, D. 1975, Mémoires of the Société Royale des Sciences de Liège, 8, 369

Siess, L. 2006, A\&A, 448, 717

Sneden, C. A. 1973, Ph.D. Thesis, Texas Univ., Austin

Sneden, C., \& Pilachowski, C. A. 1984, PASP, 96, 38

Sneden, C., Lambert, D. L., Tomkin, J., \& Peterson, R. C. 1978, ApJ, 222, 585

Takeda, Y., Sato, B., \& Murata, D. 2008, PASJ, 60, 781

Tomkin, J., Sneden, C., \& Cottrell, P. L. 1984, PASP, 96, 609

Turcotte, S., \& Richard, O. 2003, Ap\&SS, 284, 225

Ulrich, R. K. 1972, ApJ, 172, 165

van Leeuwen, F. 2007, HipPARcos, the New Reduction of the Raw Data, Astrophys. Space Sci. Lib., 350

Wu, Y., Singh, H. P., Prugniel, P., Gupta, R., \& Koleva, M. 2011, A\&A, 525, A71 